\title{
Optical nanotomography of anisotropic fluids
}

\author{
ANTONIO DE LUCA ${ }^{1 *}$, VALENTIN BARNA2*, TIMOTHY J. ATHERTON ${ }^{3}$, GIOVANNI CARBONE ${ }^{1}$, \\ MATTHEW E. SOUSA ${ }^{4}$ AND CHARLES ROSENBLATT ${ }^{3 \dagger}$ \\ ${ }^{1}$ CNR-INFM LiCryL, Cemif.Cal, c/o Physics Department, University of Calabria, 87036 Rende (CS), Italy \\ ${ }^{2}$ Faculty of Physics, University of Bucharest, PO Box Mg-11, 077125, Bucharest, Magurele, Romania \\ ${ }^{3}$ Department of Physics, Case Western Reserve University, Cleveland, Ohio 44106, USA \\ ${ }^{4} 3 \mathrm{M}$ Corporation, St. Paul, Minnesota 55144, USA \\ * These authors contributed equally to this work \\ †e-mail: rosenblatt@case.edu
}

The physical properties of anisotropic fluids can be manipulated on very short length scales of $100 \mathrm{~nm}$ or less by appropriate treatment of the confining substrate $(s)^{1,2}$. This facilitates the use of ordered fluids in a variety of scientific endeavours and applications. Although future advances will require a complete understanding of their structure at the nanoscale level, high-resolution three-dimensional optical imaging of the fluid's molecular orientation profile is beyond the reach of extant techniques ${ }^{3}$. Here, we report a powerful imaging approach based on the collection of transmitted light in the far field that is emitted from a light source with a subwavelength aperture. We acquire high-resolution images by raster-scanning, at multiple heights, an optical fibre immersed inside a thin layer of anisotropic fluid, thereby facilitating the visualization of the fluid's structure with a resolvable volume $\sim 1 / 500$ of that attainable by current methods. This novel technique offers the intriguing possibility of three-dimensional nanoscale reconstruction of a variety of soft materials, here the first direct visualization and measurement of the liquid-crystal molecular orientation relaxation length.

The idea of using a subwavelength metal-coated fibre aperture to investigate a surface with high resolution was proposed initially by Synge ${ }^{4}$; this has evolved into the technique of near-field optical microscopy ${ }^{5}$ for two-dimensional (2D) imaging. Here, we present an entirely new imaging approach that involves the use of polarized light, emitted from a tapered optical fibre immersed in an anisotropic medium, in the far field to investigate molecular orientation in three dimensions at nanoscale levels. As there are no significant scattering sources due to dielectric inhomogeneities, the near-field light does not scatter in the customary manner, but instead decays exponentially with distance and is not detected downstream. Instead, the light that reaches the detector from the fibre aperture consists of small-wave-vector Fourier components, and is retarded by a phase $\delta$ as it propagates through the continuous birefringent fluid medium. By carrying out in-plane $(x y)$ scans inside the sample at a series of heights $z_{i}$ above the surface, we obtain intensity matrix slices, from which we extract information about the fluid's local optical properties. The initial image $(i=1)$ is acquired by raster-scanning the fibre at height $z_{1}=h_{1}$ (Fig. 1) close to the nanopatterned polymer alignment layer. If $z_{1}$ is
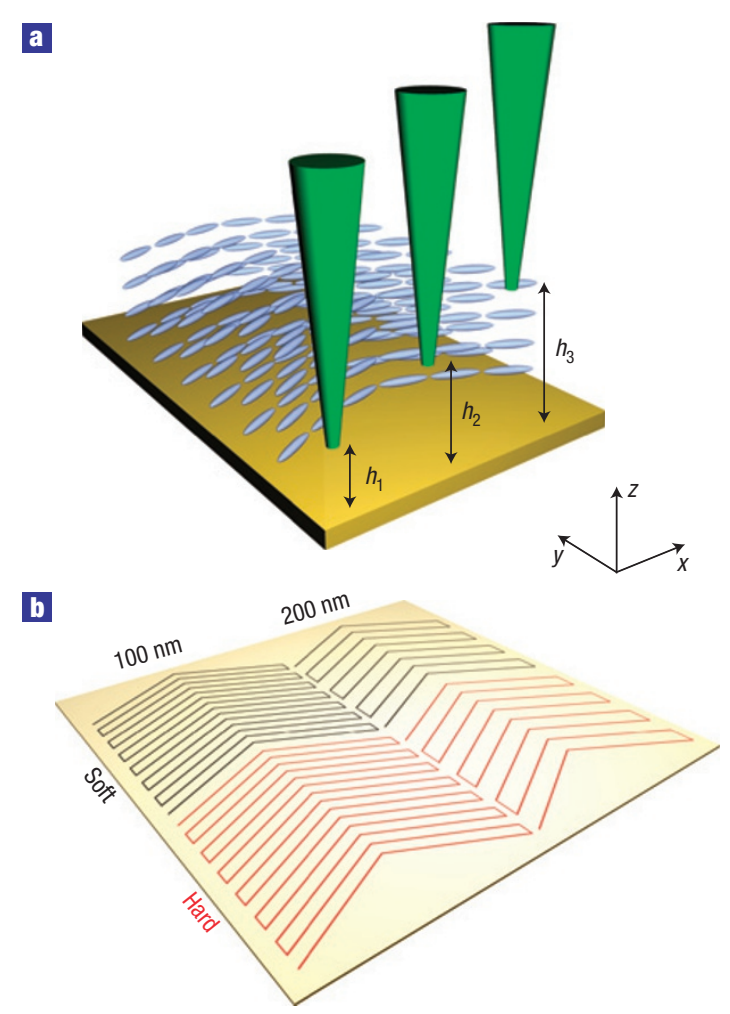

Figure 1 Schematic diagram of optical nanotomography scheme and scribing pattern on substrate. a, Schematic representation of a thin layer of an anisotropic fluid sitting atop a patterned polymer-coated substrate. The aperture of the tapered fibre is shown at three representative heights $z=h_{1}, h_{2}$ and $h_{3}$ inside the fluid. The fibre is raster-scanned at each height, illuminating the fluid below with polarized light. The transmitted light is collected and an intensity matrix / versus $x, y$ is obtained at each height. $\mathbf{b}$, Schematic representation of the scribing pattern for two different scribing strengths ( 2.1 and $5.4 \mu \mathrm{N}$ of force applied by the stylus) and at two different spacings. Each region of uniform scribing orientation corresponds to a pixel; four pixels are shown, with the pixel boundaries corresponding to the locus of points parallel to the $y$ axis at which an abrupt change in the scribing direction occurs. 
sufficiently small, the fluid's molecular orientation profile will correspond to that at the substrate, and will be nearly spatially uniform through the thickness of the slice. Each subsequent image obtained at larger $z_{i}$ contains information about all slices $1,2, \ldots$, $i-1, i$. As an example, we examine a nematic liquid crystal, the molecular orientation of which is controlled by a nanoscopic pattern scribed into the underlying polymer-coated substrate. We have selected this system as an ideal test bed for validation of our high-resolution imaging technique because there exist theoretical predictions about liquid crystal's behaviour, even though extant experimental techniques are unable to examine these small length scales. We empirically find that: (1) the lateral resolution of the fluid's optical properties is as small as $100 \mathrm{~nm}$ and vertical resolution a few tens of nanometres, and (2) the fibre's perturbation of the fluid structure is small and does not significantly affect the signal. As a consequence, we are able to observe directly the length scale of $\sim 200 \mathrm{~nm}$ over which the molecular orientation relaxes owing to the liquid crystal's elastic forces, thus demonstrating the utility of polarized optical nanotomography (ONT) for 3D imaging of soft matter.

The nematic liquid-crystal phase is characterized by long-range orientational order of the molecules ${ }^{6}$. The axis of alignment is known as the 'director', and can be manipulated azimuthally $(\varphi)$ by patterning a polymer-coated substrate on length scales as small as $100 \mathrm{~nm}$ (refs 1,2,7,8). A critical issue for numerous technologies is the tendency of the liquid-crystal director to follow the imposed pattern, which is known as the 'easy axis', and how spatially rapidly the distortion relaxes. Here, we exploit ONT to visualize in 3D the director profile on the small length scales that are characteristic of orientational relaxation.

The driving force behind this orientational homogenization is the nematic liquid-crystal's elasticity ${ }^{6}$, which favours a spatially uniform state. An underlying pattern (Fig. 1b) imposes a spatial variation on the liquid-crystal director, which relaxes azimuthally both in the substrate plane and with increasing height above the substrate. The 'extrapolation length' $L$ is the characteristic distance over which the director relaxes to a uniform state from a discontinuity in the boundary condition ${ }^{6,9}$. Quantitatively, the lowest-order anisotropic part of the interfacial free energy is given by $F=1 / 2 W_{2} \Delta \varphi^{2}$ (ref. 10), where $\Delta \varphi$ is the angular deviation from the patterned easy axis and $W_{2}$ is the quadratic 'anchoring strength coefficient'. The extrapolation length $L$ is given by $K_{i i} / W_{2}$, where $K_{i i}$ corresponds to the appropriate elastic constant $K_{11}$ for splay, $K_{22}$ for twist and $K_{33}$ for bend distortions. For typical values of $K_{i i} \sim 10^{-11} \mathrm{~N}$ and $W_{2} \sim 10^{-4}-10^{-5} \mathrm{~N} \mathrm{~m}^{-1}$ (refs 6,9), we expect that $L \sim 100-1,000 \mathrm{~nm}$, a distance too small to image by extant methods. Instead, we turn to optical nanotomography, a technique that exploits the propagating far field, for 3D imaging. (In contrast, we note that the very different approach of near-field optical microscopy (ref. 5), which involves scattering of evanescent (near-field) modes as opposed to the propagation and phase retardation associated with the ONT technique, has been used to carry out $2 \mathrm{D}$ imaging of liquid crystals ${ }^{11-15}$.)

To promote an easy-axis pattern, we scribed a polymer-coated substrate with the stylus of an atomic force microscope ${ }^{2}$ (AFM) in a herringbone configuration (Fig. 1b). Both hard and soft scribing were used and, for each of the two scribing strengths, we used two different line spacings: 100 and $200 \mathrm{~nm}$. Details can be found in Supplementary Information, Methods. Figure 2 shows a sequence of images obtained at several heights above the substrate. Qualitatively, several features are apparent. First, images obtained closer to the substrate are noisier, as would be expected from the weaker signals associated with smaller optical phase retardation $\delta$. A second observation is the appearance of 'fingers' spaced at $200 \mathrm{~nm}$

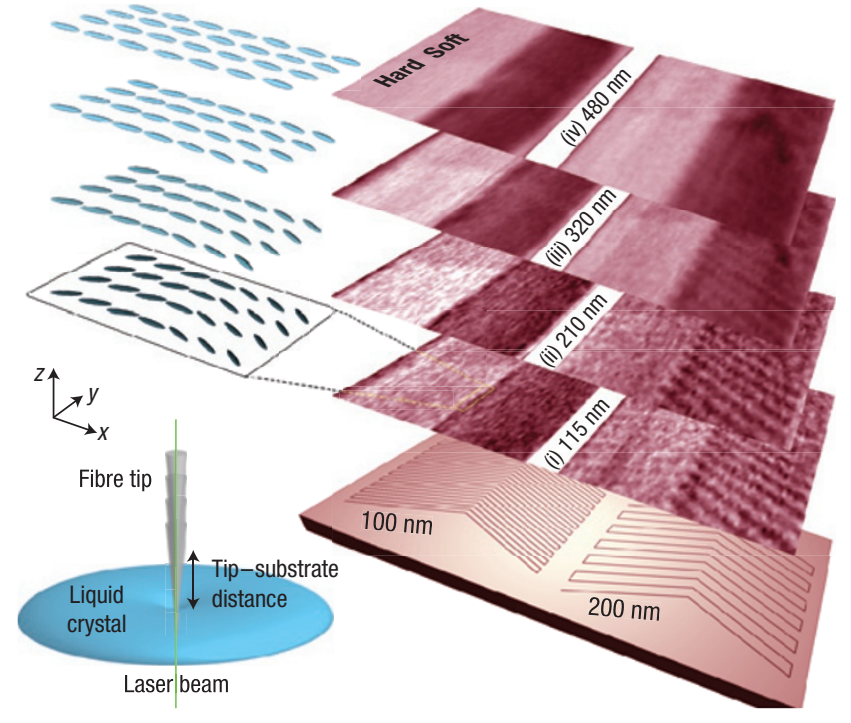

Figure 2 ONT images at different heights. A series of images created from the intensity data matrix collected at heights (i) $115 \mathrm{~nm}$, (ii) $210 \mathrm{~nm}$, (iii) $320 \mathrm{~nm}$ and (iv) $480 \mathrm{~nm}$ above the polymer-coated substrate, with an uncertainty in height of $\pm 10 \mathrm{~nm}$. The scan dimension is approximately $15 \times 9 \mu \mathrm{m}$. The polarizer makes an angle $\beta$ nominally equal to $7.5^{\circ}$ and $37.5^{\circ}$ with respect to the two easy axes.

within each of the horizontal stripes that was scribed with a spacing of $200 \mathrm{~nm}$; thus, the fingers correspond to individual passes-back and forth-of the AFM stylus. The fingers are due to an inherent asymmetry in the scribing process-the stylus is not conical and the cantilever pushes in one finger and pulls in the adjacent finger, resulting in slightly different groove depths $\Delta z$. Observations that: (1) the grooves remain distinct, and (2) the value $\Delta z=(17 \pm 3) \mathrm{nm}$ obtained from the ONT intensity contrast due to the difference in $\delta$ from one finger to the next is the same as that obtained by an AFM measurement of the topography ${ }^{16}$ indicate that excellent optical resolution is achievable, even out to $z \sim 500 \mathrm{~nm}$. A further discussion of the optical resolution can be found in Supplementary Information, Discussion.

We now examine the probe's influence on the nematic phase. Tadokoro et al. observed that the cladded fibre has negligible influence on the director orientation in the nematic phase ${ }^{11,12}$, indicating extremely weak anchoring conditions at the fibre. Our own measurements agree with their results. We examined the fibre tip inside two liquid-crystal cells of thickness $300 \mu \mathrm{m}$, one treated for planar and one for vertical alignment, finding no observable perturbation of the liquid-crystal director. This is discussed in detail in Supplementary Information, Discussion. These observations suggest that the fibre's orientational effects are small and will not perturb the director significantly. We also investigated the consequences of the fibre's motion through the liquid crystal. Images collected from forward and reverse scans are equivalent in detail, thus indicating that the liquid-crystal orientation is largely unaffected by (or very quickly relaxes back to equilibrium after) the fibre's very slow translational motion of $\sim 7 \mu \mathrm{m} \mathrm{s}^{-1}$. See Supplementary Information, Methods.

We will concentrate our data analysis on regions that are scribed with a line spacing of $100 \mathrm{~nm}$, the images of which appear nearly uniform even for the closest approach of the fibre to the substrate; detailed analysis of the images associated with the $200 \mathrm{~nm}$ line spacing will be considered elsewhere ${ }^{16}$. Data were collected at 17 individual heights $z$. Figure 3 shows 


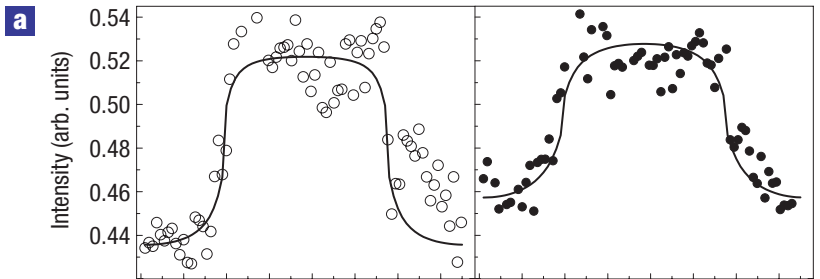

b

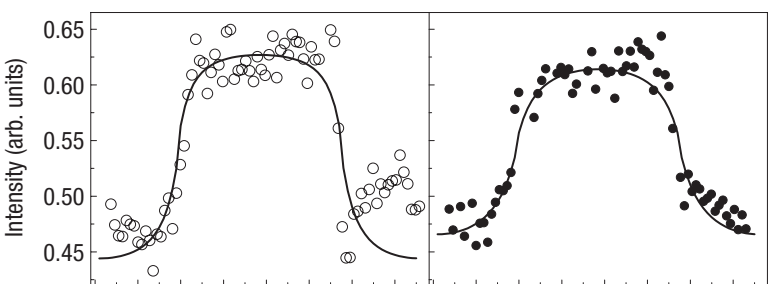

c

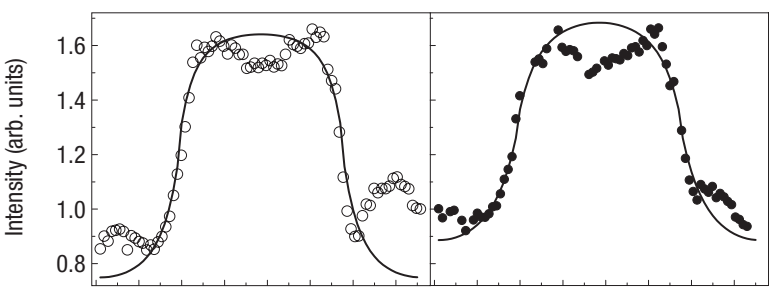

d

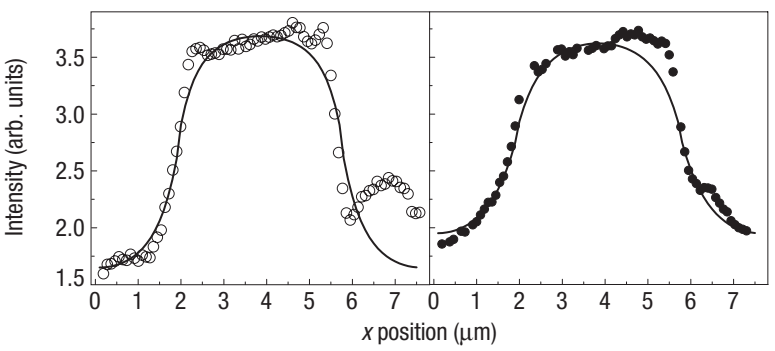

Figure 3 Data and theory for optical intensities. a-d, Experimental data and theoretical plots at four heights above the substrate: height $z$ is equal to $115 \mathrm{~nm}(\mathbf{a})$, $210 \mathrm{~nm}$ (b), $320 \mathrm{~nm}$ (c) and $480 \mathrm{~nm}$ (d). These correspond to images (i), (ii), (iii) and (iv), respectively, in Fig. 2. Results for hard-scribed substrates are plotted on the left, and for soft-scribed substrates on the right. Circles represent experimental data and lines represent calculations for the intensity based on the theoretical calculation for $\varphi(x, y, z)$ in the continuum limit. Note that the intensity scale is in arbitrary units, but is the same in all four panels.

intensity profiles for hard- (left) and soft- (right) scribed regions at heights $z=115$ (the closest approach for which the instrument could be stabilized properly), 210, 320 and $480 \mathrm{~nm}$ above the substrate. The profiles represent convolutions of the 'bare' intensity profile with the instrument function, which is due mainly to the small spread of light as it passes from the fibre's aperture to the substrate. The solid lines correspond to calculations for hard and soft scribing, which will be discussed below. We observe that the width $w$ of the region over which the intensity changes from $I_{\min }+(1 / e)(\Delta I / 2)$ to $I_{\min }+(\Delta I / 2)$ (or equivalently, from $I_{\max }-(\Delta I / 2)$ to $\left.I_{\max }-(1 / e)(\Delta I / 2)\right)$, is smaller for the hard-scribed region. Here, $I_{\min }$ and $I_{\max }$ are the minimum and maximum intensities, $\Delta I=I_{\max }-I_{\min }$ and $e$ is Euler's number $(2.71828 \ldots)$. As the polarization was chosen so that $I$ is approximately proportional to $\varphi$ close to the substrate, for the data collected at $z_{1}=115 \mathrm{~nm}$ (Fig. 3a) we can treat $w$ as an upper limit of the extrapolation length $L$ at the substrate, that is, the characteristic distance over which the director approaches

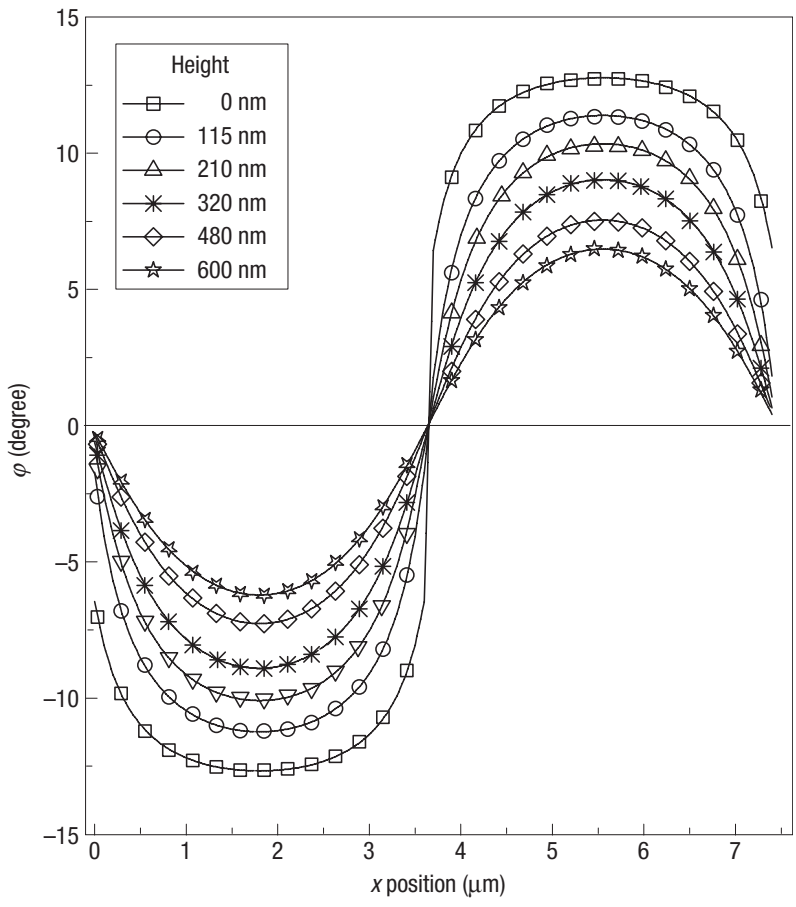

Figure 4 Theoretical azimuthal orientations $\varphi$. Theoretical calculation based on continuum theory for the azimuthal director $\varphi(x)$ at the substrate $(z=0)$ and at five other heights $z=115 \mathrm{~nm}, 210 \mathrm{~nm}, 320 \mathrm{~nm}, 480 \mathrm{~nm}$ and $600 \mathrm{~nm}$ above the substrate. Anchoring strength coefficient $W_{\varphi}=8 \times 10^{-2} \mathrm{dyn} \mathrm{cm}^{-1}$ for the calculation, corresponding to hard scribing.

its limiting value of $\varphi= \pm 15^{\circ}$ in the interior of the pixel from $\varphi=0$ at the pixel boundary. That the measured value $w$ is an upper limit for $L$ is because elasticity has already partially relaxed the director profile with increasing height and because the data reflect a convolution of the director profile with the instrument function. Nevertheless, the image in Fig. 2(i) and corresponding data in Fig. 3a represent the first direct visualization of the director relaxation. From the data in Fig. 3a, we find that $w \approx(220 \pm 70) \mathrm{nm}$ for hard scribing and $w \approx(400 \pm 140) \mathrm{nm}$ for soft scribing. Taking $K_{33} \sim 1 \times 10^{-11} \mathrm{~N}$ (ref. 6), we obtain lower limits for the azimuthal anchoring strength (recall that $W_{2} \propto 1 / L$ ) $W_{2}=(4.5 \pm 1.5) \times 10^{-5} \mathrm{~N} \mathrm{~m}^{-1}$ and $(2.5 \pm 0.8) \times 10^{-5} \mathrm{~N} \mathrm{~m}^{-1}$ for the hard- and soft-scribed regions, respectively. These values of $W_{2}$ are comparable to those obtained from indirect measurements associated with AFM-scribed polyimides ${ }^{17,18}$.

For images recorded at larger $z$, we see that the width $w$ increases with $z$, owing to the twist elasticity- (along $z$ ) and bend elasticity- (along $y$ ) induced uniformation of the director orientation with increasing $z$. Well above the substrate, the width $w$ can no longer be associated with the relaxation of the director orientation at that height. Rather, the intensity at each position $x, z$ represents the light propagation through a birefringent medium, the optic axis of which is varying in space.

To better interpret our measurements, we calculated the director orientation in the continuum approximation by minimizing the two-dimensional elastic free energy subject to the boundary condition at the interface in which the elastic torque balances the surface anchoring torque ${ }^{6}$. Details are presented in Supplementary Information, Methods. Figure 4 shows the orientation $\varphi$ versus $x$ at several heights $z$ for anchoring strength $W_{2}=8 \times 10^{-5} \mathrm{~N} \mathrm{~m}^{-1}$. Notice that the sharpness of the $\varphi$ curves 
decreases rapidly with increasing $z$, that for larger values of $z$ the amplitude of $\varphi$ decreases with increasing $z$ and that by $z \sim 320 \mathrm{~nm}$, $\varphi$ varies nearly sinusoidally with $x$.

To calculate the expected light intensity, we adopt the extremely simple approximation that the collected light has propagated through the birefringent liquid crystal as would a plane wave; this is discussed in Supplementary Information, Discussion. We then carried out a Berreman $4 \times 4$ optical calculation ${ }^{19}$ for light propagation described by our calculations for $\varphi(y, z)$ (see Supplementary Information, Methods.) In this calculation, the liquid crystal is divided into thin layers and both electric and magnetic field boundary conditions are applied at each interface, thus accounting for multiple reflections. The results are shown by the solid lines in Fig. 3. Agreement with the measured intensities at the various heights is superb. It is clear that the intensities measured at a given height $z$ do not wash out as rapidly as the azimuthal orientation $\varphi$ (Fig. 4) at that height, but rather reflect the transmission of light through the thickness between the substrate and the height of the aperture.

The results presented herein clearly demonstrate the excellent spatial resolution associated with optical nanotomography. In particular, we have visualized the extrapolation length for different anchoring conditions and have shown that our optical measurements are consistent with continuum theory. With improved signal-to-noise ratio and a more robust optical model, a fully 3D director profile reconstruction will be possible. Comparing ONT with other imaging techniques such as coherent anti-Stokes Raman scattering ${ }^{20,21}$ and fluorescence confocal polarizing microscopy ${ }^{22,23}$, ONT's strength lies in its ultrahigh resolution within $\sim 500 \mathrm{~nm}$ of the surface, the non-necessity of doping the sample with a dye and the ability to study high-birefringence materials. Of course, ONT has two potential drawbacks: it requires a free upper surface and the fibre can be invasive, depending on the material being studied. Nevertheless, for those applications amenable to ONT, its resolution is unsurpassed, being well below the diffraction limit. In summary, we have established that optical nanotomography is an extremely powerful tool for 3D imaging of liquid crystals, and also may be used for nanoscale reconstruction of a variety of select soft systems such as stacked lipid bilayers, lyotropic liquid crystals composed of macromolecules such as DNA or tobacco mosaic virus and suspensions of carbon nanotubes.

Received 15 February 2008; accepted 19 August 2008; published 21 September 2008.
References

1. Pidduck, A. J., Haslam, S. D., Bryan-Brown, G. P., Bannister, R. \& Kitely, I. D. Control of liquid crysta alignment by polyimide surface modification using atomic force microscopy. Appl. Phys. Lett. 71, 2907-2909 (1997)

2. Wen, B, Mahajan, M. P. \& Rosenblatt, C. Ultrahigh-resolution liquid crystal display with gray scale. Appl. Phys. Lett. 76, 1240-1242 (2000).

3. Pawley, J. B. Handbook of Biological Confocal Microscopy (Springer, New York, 2006).

4. Synge, E. A. A suggested method for extending microscopic resolution into the ultra-microscopic region. Phil. Mag. 6, 356-362 (1928)

5. Dunn, R. C. Near field scanning optical microscopy. Chem. Rev. 99, 2891-2927 (1999).

6. DeGennes, P. G. \& Prost, J. The Physics of Liquid Crystals (Clarendon, Oxford, 1994).

7. Kim, J.-H., Yoneya, M. \& Yokoyama, H. Tristable nematic liquid-crystal device using micropatterned surface alignment. Nature 420, 159-162 (2002).

8. Scharf, T. et al. Liquid crystal alignment on chemical nanopatterns: Control over azimuthal and polar alignment. Mol. Cryst. Liq. Cryst. 438, 1619-1629 (2005).

9. Jérôme, B. Surface effects and anchoring in liquid crystals. Rep. Prog. Phys. 54, 391-452 (1991).

10. Rapini, A. \& Papoular, M. Distortion d'une lamelle nematique sous champ magnetique conditions d'ancrage aux parois. J. Phys. (Paris) Colloq. 30, C4-54-C4-56 (1969).

11. Tadokoro, T., Saiki, T. \& Toriumi, H. Design and implementation of near field scanning optical microscope for observation of interfacial liquid crystal orientation. Jpn. J. Appl. Phys. 41 (part 2A), L152-L154 (2002)

12. Tadokoro, T., Saiki, T. \& Toriumi, H. Two dimensional analysis of liquid crystal orientation at in-plane switching substrate surface using near field scanning optical microscope. Jpn. J. Appl. Phys. 42 (part 2), L57-L59 (2003).

13. Huser, T., Lacoste, T., Heinzelmann, H. \& Kitzerow, H. S. Scanning near-field optical microscopy of cholesteric liquid crystals. J. Chem. Phys. 108, 7876-7880 (1998).

14. Moyer, P. J., Kämmer, S., Walzer, K. \& Hietschold, M. Investigations of liquid crystals and liquid ambients using near field scanning optical microscopy. Ultramicroscopy 61, 291-294 (1995).

15. Higgins, D. A., Hall, J. E. \& Xie, A. Optical microscopy studies of dynamics within individual polymer-dispersed liquid crystal droplets. Acc. Chem. Res. 38, 137-145 (2005).

16. Barna, V., De Luca, A. \& Rosenblatt, C. Nanoscale alignment and optical nanoimaging of a birefringent liquid. Nanotechnology 19, 325709 (2008).

17. Wen, B. \& Rosenblatt, C. Planar nematic anchoring due to a periodic surface potential. J. Appl. Phys. 89, 4747-4751 (2001).

18. Wang, R., Syed, I. M., Carbone, G., Petschek, R. G. \& Rosenblatt, C. Bend-induced melting of the smectic-A phase: Analogy to a type-I superconductor. Phys. Rev. Lett. 97, 167802 (2006).

19. Berreman, D. W. Optics in stratified and anisotropic media: 4X4-matrix formulation. J. Opt. Soc. Am. 62, 503-510 (1972).

20. Kachynski, A. V., Kuzmin, A. N., Prasad, P. N. \& Smalyukh, I. I. Coherent anti-Stokes Raman scattering polarized microscopy of three-dimensional director structures in liquid crystals. Appl. Phys. Lett. 91, 151905 (2007).

21. Saar, B. G., Park, H.-S., Xie, X. S. \& Lavrentovich, O. D. Three-dimensional imaging of chemical bond orientation in liquid crystals by coherent anti-Stokes Raman scattering microscopy. Opt. Express 15 13585 (2007).

22. Pawley, J. B. Handbook of Biological Confocal Microscopy 2nd edn (Plenum, New York, 1995).

23. Smalyukh, I. I., Shiyanovskii, S. V. \& Lavrentovich, O. D. Three dimensional imaging of orientational order by fluorescence confocal polarizing microscopy. Chem. Phys. Lett. 336, 88 (2001).

Supplementary Information accompanies the paper at www.nature.com/naturephysics.

\section{Acknowledgements}

This work was supported by the US Department of Energy's Basic Energy Science programme and the Petroleum Research Fund of the American Chemical Society. We are grateful to J.-H. Lee for assistance.

Author contributions

Project conception (C.R.), planning and experimental implementation (A.D.L., V.B., G.C., M.E.S., C.R.), data analysis (A.D.L., V.B.), theoretical modelling (T.J.A.) and overall integration of experiment and theory (A.D.L., V.B., T.J.A., G.C., C.R.).

\section{Author information}

Reprints and permissions information is available online at http://npg.nature.com/reprintsandpermissions. Correspondence and requests for materials should be addressed to C.R. 